\title{
Transferrin-PEG-PE modified dexamethasone conjugated cationic lipid carrier mediated gene delivery system for tumor-targeted transfection
}

This article was published in the following Dove Press journal:

International Journal of Nanomedicine

18 May 2012

Number of times this article has been viewed

Wei Wang'

Fang Zhou ${ }^{2}$

Linfu $\mathrm{Ge}^{2}$

Ximin Liu $^{2}$

Fansheng Kong ${ }^{2}$

'Department of Chinese Medicine Integrated Traditional Chinese Medicine and Western Medicine, General Hospital of Ji'nan Command, Ji'nan, China; ${ }^{2}$ Department of Hematology, General Hospital of Ji'nan Command, Ji'nan, China
Correspondence: Fansheng Kong Department of Hematology, General Hospital of Ji'nan Command, PLA, 25 Shifan Road, Ji'nan, 25003I, China $\mathrm{Tel}+86053151665200$

Email kongfanshengphd@yahoo.com.cn
Background: The main barriers to non-viral gene delivery include cellular and nuclear membranes. As such, the aim of this study was to develop a type of vector that can target cells through receptor-mediated pathways and by using nuclear localization signal (NLS) to increase the nuclear uptake of genetic materials.

Methods: A dexamethasone (Dexa)-conjugated lipid was synthesized as the material of the solid lipid nanoparticles (SLNs), and transferrin (Tf) was linked onto polyethylene glycolphosphatidylethanolamine (PEG-PE) to obtain Tf-PEG-PE ligands for the surface modification of the carriers. The in vitro transfection efficiency of the novel modified vectors was evaluated in human hepatoma carcinoma cell lines, and in vivo effects were observed in an animal model. Results: Tf-PEG-PE modified SLNs/enhanced green fluorescence protein plasmid (pEGFP) had a particle size of $222 \mathrm{~nm}$ and a gene loading quantity of $90 \%$. Tf-PEG-PE-modified SLNs/ pEGFP (Tf-SLNs/pEGFP) displayed remarkably higher transfection efficiency than non-modified SLNs/pEGFP and the vectors not containing Dexa, both in vitro and in vivo.

Conclusion: It can be concluded that $\mathrm{Tf}$ and Dexa could function as an excellent active targeting ligand to improve the cell targeting and nuclear targeting ability of the carriers, and the resulting nanomedicine could be a promising active targeting drug/gene delivery system.

Keywords: gene delivery, active targeting, transferrin-PEG-PE, dexamethasone conjugated lipid, nuclear localization

\section{Introduction}

The success of gene-loaded nanomedicine requires efficient targeted gene delivery systems. Non-viral gene delivery systems, such as liposomes, ${ }^{1}$ polymers, ${ }^{2}$ and solid lipid nanoparticles (SLNs), ${ }^{3}$ have been developed as alternatives to viral gene delivery systems, in order to overcome the disadvantages of viral vectors. ${ }^{4}$ Although non-viral carriers have advantages, such as being less toxic, low immunogenic, and easy to modify, low transfection efficiency compared to viral carriers limit their applications. ${ }^{5}$ Therefore, diverse approaches have been applied to potentiate non-viral gene delivery vectors. ${ }^{6}$

The main barriers to non-viral gene delivery include cellular and nuclear membranes. ${ }^{6,7}$ Cellular uptake could be facilitated through receptor-mediated pathways by surface modification of specific ligands to carriers. ${ }^{89}$ In addition, nuclear localization signals (NLS) have been used to increase nuclear uptake of genetic materials. ${ }^{10,11}$ The glucocorticoid receptor (GR) is a nuclear receptor; ${ }^{12}$ when it binds to the ligand, the receptor-ligand complex translocates from the cytoplasm to the nucleus. ${ }^{13}$ It has been reported that glucocorticoid receptors can dilate nuclear pores up to $60 \mathrm{~nm}$ during the translocation process, which is favorable for the translocation of 
DNA into the nucleus. ${ }^{14}$ Therefore, the transport of genes into the nucleus may be enhanced by conjugating glucocorticoids to carriers. Dexamethasone (Dexa), an effective, synthetic glucocorticoid, could bind to the glucocorticoid receptor after cellular entry and translocate the complexes into the nucleus. ${ }^{15-20}$ The results of these studies have confirmed that Dexa-conjugated vector/DNA complexes could be efficiently delivered into the nucleus with the glucocorticoid receptor, resulting in increased transgene expression.

Recently, much attention has been given to the use of cationic SLNs as gene carriers that might offer a number of technological advantages, including better storage stability in comparison to liposomes, the possibility of steam sterilization and lyophilization, large scale production with qualified production lines, and the use of substances that are generally accepted as safe. ${ }^{21-26}$ The cationic lipids used for the preparation of lipid nanoparticles include cetyltrimethylammonium bromide (CTAB), ${ }^{27} \mathrm{~N}$-[1-(2,3-dioleyloxy) propyl]-N,N,Ntri-methylammonium chloride (DOTMA), ${ }^{28}$ and dimethyldioctadecylammonium bromide (DDAB). ${ }^{29}$ However, toxicity is still an obstacle to the use of cationic lipids. Some researchers have indicated that cationic lipids with ester bonds are more biodegradable and, therefore, are associated with less cytotoxicity. ${ }^{23,30,31}$ Tang and colleagues demonstrated that 6-lauroxyhexyl ornithinate (LHON) with one tail was more efficient and had lower cytotoxicity. Therefore, LHON was used as the cationic lipid in this study.

Polyethylene glycol-phosphatidylethanolamine (PEGPE) conjugates with various PEG lengths, and terminal-targeted moieties can provide extremely stable, long-circulating, and actively targeted nanocarriers, which spontaneously accumulate at specific sites. ${ }^{32,33}$ These types of ligands also have been used previously by our group for surface modification of vehicles to achieve targeted gene delivery. ${ }^{1,34}$ Transferrin (Tf), an iron-binding glycoprotein, is a well-studied ligand for delivering anticancer drugs/genes, due to the increased number of Tf receptors found on tumor cells compared to normal cells. ${ }^{35,36}$ This receptor-mediated endocytosis could facilitate the delivery of drugs/genes into cells. ${ }^{37-39}$ In this study, Tf was linked onto PEG-PE to form Tf-PEG-PE as ligands for the surface modification of nanocarriers.

In the present study, a Dexa-conjugated LHON (Dexa-LHON) was synthesized for the enhancement of nuclear localization of a gene. Enhanced green fluorescence protein plasmid ( $\mathrm{pEGFP}$ ) was used as the model gene. After the preparation of gene-loaded cationic SLNs (SLNs/pEGFP), novel Tf-PEG-PE was synthesized and modified onto the surface of the SLNs/pEGFP. The in vitro transfection efficiency of the novel modified vectors was evaluated in human hepatoma carcinoma cell lines (HepG2 cells), and in vivo effects were observed in mice bearing human HepG2 cells model. Unmodified SLNs/ pEGFP, modified SLNs not containing Dexa (Tf-non-DexaSLNs/pEGFP), and unmodified non-Dexa-SLNs/pEGFP were used as controls.

\section{Materials and methods \\ Materials}

Stearic acid, L- $\alpha$-phosphatidylethanolamine (PE), 2- iminothiolane (Traut's Reagent), Human Tf (iron-free), and (3-[4,5-dimehyl-2-thiazolyl]-2,5-diphenyl-2H-tetrazolium bromide (MTT) were purchased from Sigma-Aldrich Co, Ltd (St Louis, MO). Injectable soya lecithin was obtained from Shanghai Taiwei Pharmaceutical Co, Ltd (China). Dexamethasone-21- mesylate was purchased from Steraloids Inc. LHON was synthesized according to a previous procedure. ${ }^{30}$ Maleimide- $\mathrm{PEG}_{2000}-\mathrm{COOH}$ was purchased from Shanghai Yare Biotech Inc, (Shanghai, China). pEGFP-N1 was provided by Shandong University (Shandong, China). Quant-iT ${ }^{\mathrm{TM}}$ PicoGreen ${ }^{\circledR}$ dsDNA quantitation reagent was obtained from Invitrogen by Life Technologies (Carlsbad, CA). HepG2 cells were obtained from the American type culture collection (Manassas, VA). All other chemicals were of analytical grade or higher.

\section{Animals}

BALB/c mice (4-6 weeks old, 18-22 g) were purchased from the Medical Animal Test Center of Shandong Province and housed under standard laboratory conditions. All animal experiments complied with the requirements of the National Act on the Use of Experimental Animals (People's Republic of China).

\section{Synthesis of Dexa-LHON and Tf-PEG-PE}

The Dexa-LHON conjugation reaction was performed using the method reported previously, with some modification. ${ }^{6}$ The synthesis scheme is depicted in Figure 1. Briefly, Dexa coupling to LHON was performed with 2 equivalent of Traut's reagent, and 2 equivalent of dexamethasone mesylate in anhydrous DMSO for 6 hours at room temperature (RT). The same volume of water was added to the reaction mixture, and it was dialyzed against pure water and filtered through a $0.45 \mu \mathrm{m}$, syringe-type filter, to remove insoluble impurities. Then, the product was obtained after freeze-drying, yielding a white, crystalline powder. 


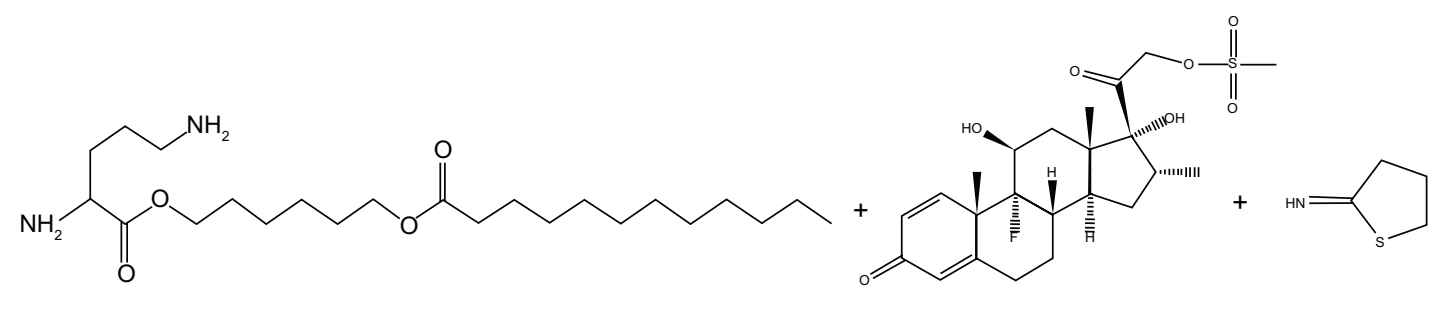

LHON

Dexa

Traut's reagent

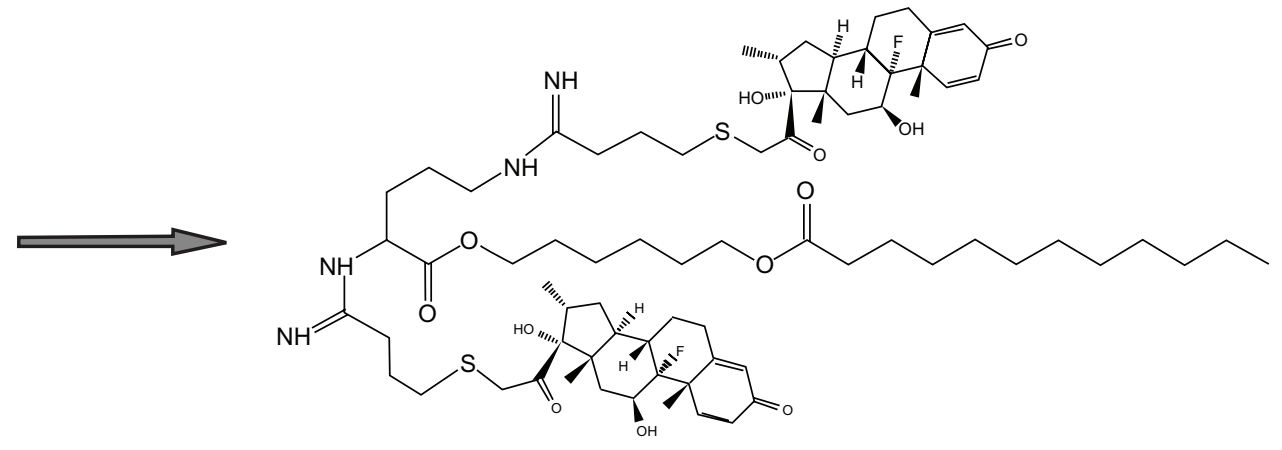

Dexa-LHON

Figure I General reaction scheme for synthesis of Dexa-LHON.

Abbreviations: Dexa, Dexamethasone; LHON, 6-lauroxyhexyl ornithinate.

Tf-PEG-PE ligands were synthesized as described in Figure 2. Maleimide- $\mathrm{PEG}_{2000}-\mathrm{COOH}(100 \mathrm{mg})$ was dissolved with dimethyl sulfoxide (DMSO) and stirred with PE (36 mg) as a mixture. 1-[3-(dimethylamino)propyl]3-ethylcarbodiimide $(\mathrm{EDC} \cdot \mathrm{HCl})(72 \mathrm{mg})$ and triethylamine (TEA, 1 equivalent of EDC $\cdot \mathrm{HCl}$ ) were dissolved in DMSO, added, dropwise into the mixture in an ice bath, and stirred for 36 hours, to produce Maleimide-PEG-CO-NH-PE. Tf was first modified with 1 equivalent of Traut's reagent to complete thiolation of $\mathrm{Tf}^{40}{ }^{4}$ The thiolated $\mathrm{Tf}$ was then added to the Maleimide- $\mathrm{PEG}_{2000}-\mathrm{COOH}$ solution, and the mixture was incubated for 2 hours at room temperature, with gentle stirring. The product was dialyzed against Milli-Q water for 24 hours to form Tf-PEG-PE solution. The mixture was centrifuged at $10,000 \mathrm{~g}$ for $30 \mathrm{~min}$ at $4{ }^{\circ} \mathrm{C}$, and then resuspended in PBS (pH 7.4).

\section{Preparation of cationic SLNs/pEGFP}

SLNs were prepared following the nanoprecipitation method (solvent displacement technique), as described previously (Figure 3) ${ }^{2,34}$ Stearic acid (50 mg) and injectable soya lecithin $(30 \mathrm{mg})$ were accurately weighted and dissolved in $10 \mathrm{~mL}$ acetone. The organic phase was added, dropwise, into the $0.1 \%$ Dexa-LHON solution, which was being stirred at $600 \mathrm{rpm}$ at room temperature. When complete evaporation of the organic solvent occurred, the redundant stabilizers were separated by ultracentrifugation at $1000 \mathrm{~g}, 4^{\circ} \mathrm{C}$, for $20 \mathrm{~min}$. The pellet was vortexed and resuspended in Milli-Q water, washed three times, filtered through a $0.45 \mu \mathrm{m}$ membrane, and adjusted to $\mathrm{pH} 7.0 \pm 0.1$ with sodium hydroxide. The obtained SLN suspensions were stored at $2{ }^{\circ} \mathrm{C}-8^{\circ} \mathrm{C}$. Non-Dexa-SLNs were prepared in the same manner, but using LHON without Dexa.

$$
\text { Maleimide-PEG-COOH + PE } \underset{\text { DMSO }}{\text { EDC } \cdot \mathrm{HCI}, \mathrm{TEA}} \text { \ Maleimide-PEG-CO-NH-PE }
$$

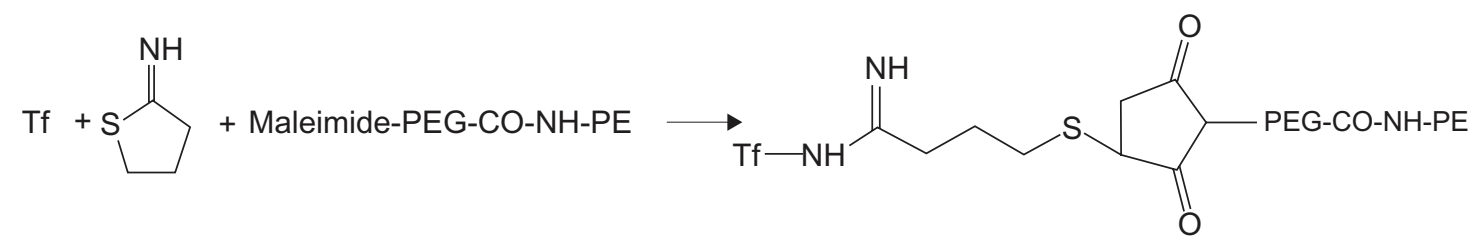

Figure 2 General reaction scheme for synthesis of Tf-PEG-PE.

Abbreviations: DMSO, dimethylsulfoxide; PEG, polyethylene glycol; PE, L- $\alpha$-phosphatidylethanolamine; TEA, triethylamine; Tf, transferrin. 


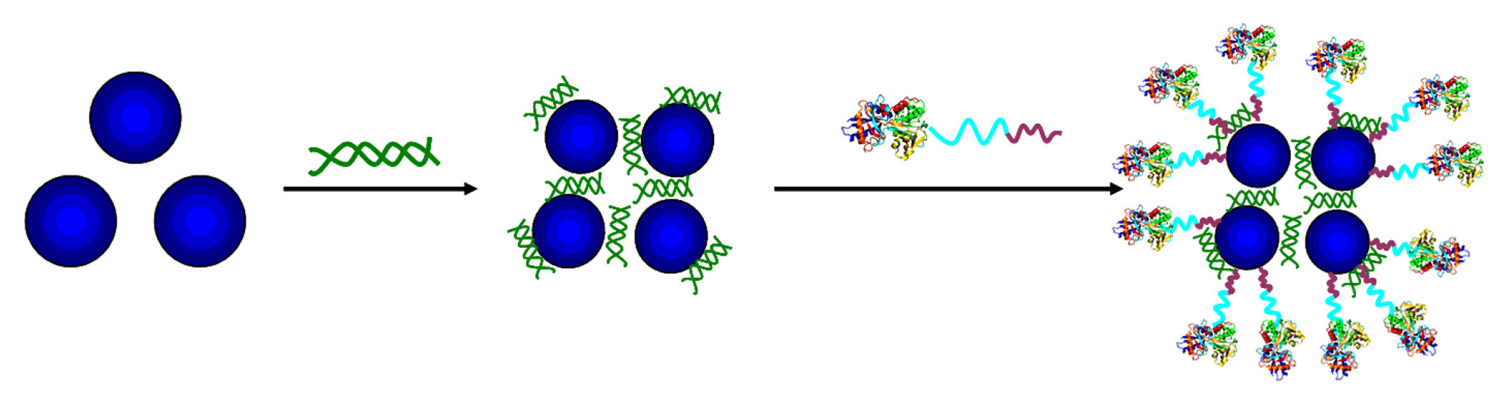

\section{SLNs $\quad$ pEGFP SLNs/pEGFP Tf-PEG-PE Tf-SLNs/pEGFP}

Figure 3 Preparation of Tf-PEG-PE-modified SLNs/pEGFP.

Abbreviations: SLNs, solid lipid nanoparticles; pEGFP, enhanced green fluorescence protein plasmid; Tf, transferrin; PEG, polyethylene glycol; PE, L- $\alpha$-phosphatidylethanolamine.

SLNs/pEGFP (non-Dexa-SLNs/pEGFP) complexes were prepared by incubating the SLNs (non-Dexa-SLNs) with pEGFP. Briefly, pEGFP was mixed with SLNs by vortexing the particles with a $1 \mathrm{mg} / \mathrm{mL}$ solution of pEGFP for 20 seconds. Incubation of the mixture for 20 minutes at RT facilitated the formation of SLNs/pEGFP (non-Dexa-SLNs/ pEGFP).

\section{Modification of SLNs/pEGFP with Tf-PEG-PE}

The Tf-PEG-PE ligands were dissolved in $5 \mathrm{~mL}$ of PBS (pH 7.4). Then, the solution was added, dropwise, to $20 \mathrm{~mL}$ of SLNs/pEGFP complexes that was stirred at $600 \mathrm{rpm}$ at RT, leading to the immediate modification. Subsequently, free Tf-PEG-PE was removed from modified SLNs/pEGFP by gel chromatography, using a Sephadex ${ }^{\circledR}$ G-50 column. The obtained complexes were resuspended in Milli-Q water, washed three times, and filtered through a $0.80 \mu \mathrm{m}$ pore size membrane, to obtain Tf-PEG-PE-SLNs/pEGFP (Tf-SLNs/pEGFP) (Figure 3). During this procedure, TfPEG-PE ligands were continuously coated onto the surface of the SLNs/DNA, which would cause the decrease of zeta potential. To optimize the modification ratio, Tf-PEG-PE ligands dissolved in PBS were designed at different weight ratios to the SLNs (w/w), and the zeta potential of the complexes was determined. Non-Dexa-SLNs/pEGFP were also modified per the above-mentioned method to form Tf-nonDexa-SLNs/pEGFP.

\section{Characterization of Tf-SLNs/pEGFP}

Size, size distribution, and zeta potential

The mean particle size, polydispersity index (PDI), and zeta potential of SLNs, SLNs/pEGFP, and Tf-SLNs/pEGFP were analyzed by photon correlation spectroscopy (PCS), with a Zetasizer 3000 (Malvern Instruments, Malvern, England). The average particle size was expressed as volume mean diameter, and the reported value was represented as mean $\pm \operatorname{SD}(n=3)$.

Gene loading capacity: PicoGreen ${ }^{\circledR}$ fluorometry assay During the modification procedure, Tf-PEG-PE ligands were coated onto the SLNs/pEGFP carriers, due to the electric charge and lipophilic interaction. PicoGreen fluorometry assay was used to determine the appropriate modification rate of Tf-PEG-PE ligands and to quantitate the amount of pEGFP carried by the optimum modified SLNs. ${ }^{1}$ Different ratios of Tf-PEG-PE to SLNs/pEGFP (from $2 \%$ to $50 \%$, w/w) were used to prepare Tf-SLNs/pEGFP complexes, and the pEGFP was isolated from Tf-SLNs/pEGFP by centrifugation at $1000 \mathrm{~g}, 4^{\circ} \mathrm{C}$, for 30 minutes. The concentration of pEGFP was determined by fluorescence, comparing with the supernatant from blank SLNs. The amount of pEGFP loaded into the SLNs was calculated according to the linear calibration curve of pEGFP: gene loading quantity $(\%)=($ total amount of pEGFP - amount of free pEGFP)/total amount of DNA $\times 100$.

\section{In vitro release of Tf-SLNs/pEGFP}

The in vitro release studies of SLNs/pEGFP and Tf-SLNs/ pEGFP were performed in PBS (pH 7.4). ${ }^{23}$ Aliquots of complexes were suspended in $1 \mathrm{~mL}$ of PBS, in Eppendorf ${ }^{\circledR}$ tubes, and vortexed for 30 seconds. The tubes were then placed in a $37^{\circ} \mathrm{C}$ shaking water bath $(100 \mathrm{rpm})$. Separate tubes were used for different data points. At predetermined time intervals, the suspensions were centrifuged (1000 g, 30 minutes), and the amount of DNA released in the supernatant was analyzed by PicoGreen assay (mentioned in Section 2.6.2). Background readings were obtained using the supernatants from the blank SLNs. 


\section{In vitro cytotoxicity evaluation}

To examine cytotoxicity, HepG2 cells were seeded in 96-well plates at $8 \times 10^{3}$ cells/well and incubated for 24 hours, to allow cell attachment. ${ }^{41}$ The cells were incubated with SLNs/pEGFP and Tf-SLNs/pEGFP complexes at various concentrations (10, $20,50,100$, and $200 \mu \mathrm{g} / \mathrm{mL}$ ) for 48 hours at $37^{\circ} \mathrm{C}$ and $5 \%$ $\mathrm{CO} 2$ atmosphere, respectively. Lipofectamine ${ }^{\mathrm{TM}} 2000$ (Lipo) was used as a positive control, according to the manufacturer's procedures. Cells without incubation were used as a negative control. Cellular viability was assessed using Cell Counting Kit-8 (CCK-8; Dojindo Molecular Technologies, Inc., Gaithersburg, $\mathrm{MD})$, according to the manufacturer's procedures; the absorbance at $450 \mathrm{~nm}$ was measured using a microplate reader (Model 680; BIO-RAD, USA). Cells without the addition of CCK-8 were used as a blank to calibrate the spectrophotometer to zero absorbance. The relative cell viability (\%) was calculated as:

$$
\left(\mathrm{Abs}_{\text {sample }}-\mathrm{Abs}_{\text {blank }}\right) /\left(\mathrm{Abs}_{\text {control }}-\mathrm{Abs}_{\text {blank }}\right) \times 100 .
$$

\section{In vitro transfection analysis}

For transfection efficiency analysis, the HepG2 cells were seeded into 24 -well plates at a density of $1 \times 10^{5}$ cells/well and transfected the next day at $80 \%-90 \%$ confluency. Prior to transfection, the media were replaced with $400 \mu l$ transfection media containing Tf-SLNs/pEGFP. Naked DNA, unmodified SLNs/pEGFP, non-Dexa-SLNs/pEGFP, and Tf-non-DexaSLNs/pEGFP were used as controls. The original incubation medium was replaced with $1 \mathrm{~mL}$ of complete medium after incubation at $37^{\circ} \mathrm{C}$, for 4 hours, under a $5 \% \mathrm{CO}_{2}$ atmosphere. The cells were incubated and studied until 72 hours posttransfection. To quantitate the transfection efficiency, the cells were washed with $1 \mathrm{~mL}$ of PBS $\left(100 \mathrm{~g}, 4^{\circ} \mathrm{C}\right.$, for $\left.5 \mathrm{~min}\right)$ and were detached with trypsin/EDTA. The supernatant was discarded and resuspended with $300 \mu 1$ of PBS, mixed well, and added into the flow cytometry, to determine the amount of HepG2 cells that had been successfully transfected.

\section{Tumor model preparation}

Tumor-bearing mice were prepared by inoculating (s.c.) a suspension of HepG2 cells $\left(2 \times 10^{6}\right.$ cells $)$ into the right armpit of BALB/c mice. ${ }^{40,42}$ Briefly, the mice were acclimatized at a temperature of $25^{\circ} \mathrm{C} \pm 2{ }^{\circ} \mathrm{C}$ and a relative humidity of $70 \% \pm 5 \%$ under natural light/dark conditions for one week before dosing. Then, the mice were injected subcutaneously in the right armpit with HepG2 cells suspended in PBS. Tumors were permitted to reach $8-10 \mathrm{~mm}$ in diameter before initiation of the studies.

\section{In vivo transfection studies}

In vivo transfection activity of Tf-SLNs/pEGFP was evaluated against HepG2 solid tumors in mice. Five groups of tumor-bearing mice (eight per group) were used. The mice were injected intravenously with naked DNA, SLNs/pEGFP, non-Dexa-SLNs/ pEGFP, Tf-non-Dexa-SLNs/pEGFP, and Tf-SLNs/pEGFP. The mice were then euthanized at 48 hours or 72 hours after injection, and the tumor tissue samples were removed.$^{43}$ The tumor tissues were homogenized by pressing the samples through a $30 \mu \mathrm{m}$ cell mesh with the plunger of a $10 \mathrm{~mL}$ syringe; erythrocyte lysis buffer was added during homogenization to lyse the red blood cells. The homogenates were washed three times with PBS containing $0.5 \%$ bovine serum albumin and then filtered. The cells were obtained after centrifugation $\left(4^{\circ} \mathrm{C}, 100 \mathrm{~g}\right.$, five minutes $)$ and were seeded into 24-well plates in $1 \mathrm{~mL}$ of Dulbecco's Modified Eagle's Medium with 10\% fetal bovine serum (FBS). The fluorescent cells were observed using an inversion fluorescence microscope, at which time pictures were taken for the record. After that, the cells were washed with $1 \mathrm{~mL}$ of PBS (100 g, $4^{\circ} \mathrm{C}$ for 5 minutes) and were detached with trypsin/EDTA. The supernatant was discarded, resuspended with $300 \mu \mathrm{l}$ of PBS, and added into the flow cytometry to quantitate the amount of HepG2 cells that were successfully transfected.

\section{Statistical analysis}

All studies were repeated three times, and all measurements were carried out in triplicate. Results were reported as means \pm standard deviation (SD). Statistical significance was analyzed using the Student's $t$-test. Differences between experimental groups were considered significant when the $P$-value was less than $0.05(P<0.05)$.

\section{Results}

\section{Structure confirmation of Dexa-LHON and Tf-PEG-PE}

The structures of Dexa-LHON and Tf-PEG-PE were confirmed by ${ }^{1} \mathrm{H}$ NMR spectroscopy. The ${ }^{1} \mathrm{H}$ NMR (dimethyl sulfoxide-d6, $300 \mathrm{mHz}$ ) data of Dexa-LHON is presented, and each proton peak is identified, in Figure 4. The peak intensity ratio was calculated between the ethyl protons of LHON backbone and the two methyl protons at $13^{\prime}$ and $16^{\prime}$ position of the five-numbered Dexa ring ( $\delta 0.7-1.1 \mathrm{ppm})$. From the ${ }^{1} \mathrm{H}$ NMR calculations, it was observed that $2 \mathrm{~mol}$ of Dexa was conjugated per $1.2 \mathrm{~mol}$ of LHON. The production rate was around $80 \%$.

The ${ }^{1} \mathrm{H}$ NMR data of Tf-PEG-PE (dimethyl sulfoxide$\mathrm{d} 6,300 \mathrm{mHz})$ was $\delta 2.50\left(\mathrm{CH}_{2} \mathrm{CO}, \mathrm{OCH}_{2} \mathrm{CH}_{2} \mathrm{NHCO}\right), 3.28$ 

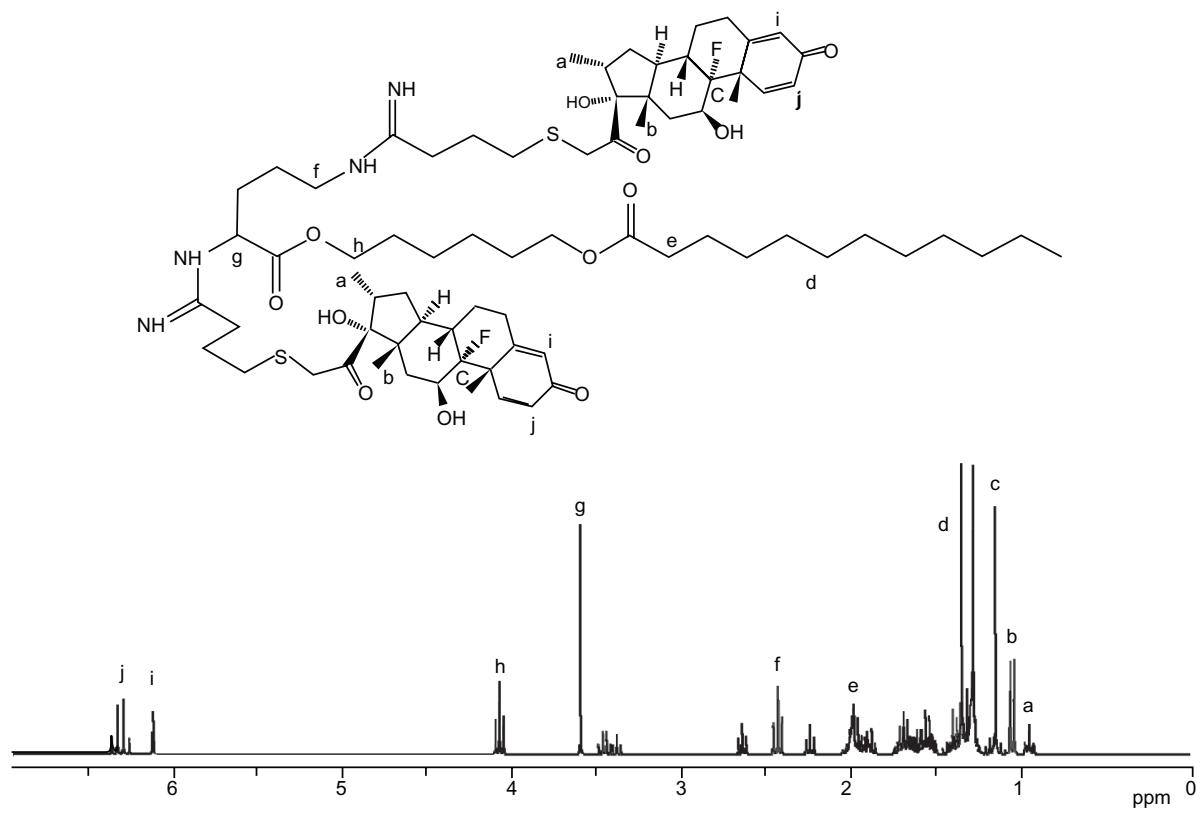

Figure 4 'H NMR spectroscopy of Dexa-LHON.

Abbreviations: Dexa, Dexamethasone; LHON, 6-lauroxyhexyl ornithinate.

$\left(\mathrm{CH}_{2} \mathrm{~N}\right), 3.36-3.48\left(\mathrm{OCH}_{2} \mathrm{CH}_{2}\right), 3.65(\mathrm{NHCO})$, and 6.08 (NH). The production rate was around $75 \%$.

\section{Optimization of the modification ratio}

Tf-PEG-PE ligands were continuously coated onto the surface of the SLNs/DNA. This procedure would mask the cationic surface charge SLNs/DNA complexes and cause the decrease of zeta potential. The best ratio of the ligands to the carriers was optimized by measuring the change of Zeta potential. As illustrated in Figure 5, the optimized ratio of Tf-PEG-PE to SLNs/DNA was $30 \%$. This ratio was determined for further experiments.

\section{Characterization of Tf-SLNs/pEGFP} Size, zeta potential, and gene-loading capacity Mean particle size, polydispersity index (PDI), and zeta potential of SLNs, SLNs/pEGFP, and Tf-SLNs/pEGFP are characterized and summarized in Table 1.

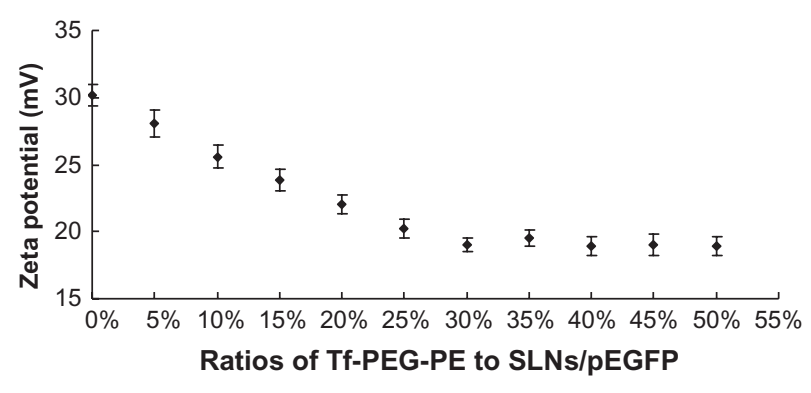

Figure 5 Optimization of the modification ratios of Tf-PEG-PE to SLNs/pEGFP. Abbreviations: Tf, transferrin; PEG, polyethylene glycol; PE, L- $\alpha-$ phosphatidylethanolamine; SLNs, solid lipid nanoparticles; pEGFP, enhanced green fluorescence protein plasmid.
In vitro release of Tf-SLNs/pEGFP

The in vitro release profiles of Tf-SLNs/pEGFP and unmodified SLNs/pEGFP are illustrated in Figure 6. Both the Tf-SLNs/pEGFP and unmodified SLNs/pEGFP reached over $80 \%$ gene release at the time point of 72 hours.

\section{In vitro cytotoxicity evaluation}

In vitro cytotoxicity of Tf-SLNs/pEGFP and SLNs/pEGFP was evaluated by CCK- 8 in HepG2 cells at different concentrations. The cell viabilities of the vectors over the studied concentration range $(10 \sim 200 \mu \mathrm{g} / \mathrm{mL})$ were between $80 \%$ and $100 \%$ compared with the controls (Figure 7).

\section{In vitro transfection analysis}

The in vitro transfection efficiencies of Tf-SLNs/pEGFP, naked DNA, unmodified SLNs/pEGFP, non-Dexa-SLNs/pEGFP, and Tf-non-Dexa-SLNs/pEGFP were evaluated in HepG2 cells after 72 hours of transfection (Figure 8). Tf-SLNs/pEGFP showed higher transfection efficiency than Tf-non-DexaSLNs/pEGFP and unmodified SLNs/pEGFP at 48 and 72 hours post-transfection $(P<0.05)$.

\section{In vivo gene delivery}

In vivo gene delivery activity of Tf-SLNs/pEGFP was evaluated against HepG2 solid tumors in mice. The in vivo transfection efficiencies of Tf-SLNs/pEGFP, naked DNA, unmodified SLNs/pEGFP, non-Dexa-SLNs/pEGFP, and Tfnon-Dexa-SLNs/pEGFP were observed and are shown in Figure 9. Tf-SLNs/pEGFP had a higher transfection efficiency at 
Table I Particle size, zeta potential, and gene loading quantity of different vectors

\begin{tabular}{lllll}
\hline $\begin{array}{l}\text { Characteristics/ } \\
\text { samples }\end{array}$ & $\begin{array}{l}\text { Mean particle } \\
\text { size }(\mathbf{n m})\end{array}$ & $\begin{array}{l}\text { Polydispersity } \\
\text { index (PDI) }\end{array}$ & $\begin{array}{l}\text { Zeta potential } \\
(\mathbf{m V})\end{array}$ & $\begin{array}{l}\text { Gene loading } \\
\text { quantity }(\%)\end{array}$ \\
\hline SLNs & $117.6 \pm 3.5$ & $0.13 \pm 0.03$ & $+42.5 \pm 2.1$ & 0 \\
SLNs/pEGFP & $178.1 \pm 3.9$ & $0.18 \pm 0.02$ & $+30.4 \pm 2.8$ & 91 \\
Tf-SLNs/pEGFP & $221.8 \pm 5.8$ & $0.14 \pm 0.02$ & $+18.9 \pm 1.9$ & 90 \\
\hline
\end{tabular}

different time intervals than the other vectors. Flow cytometry was applied to future quantitate the cells that were successfully transfected. As shown in Figure 10, Tf-SLNs/pEGFP displayed remarkably higher transfection efficiency than SLNs/pEGFP and the other vectors $(P<0.05)$.

\section{Discussion}

The aim of this study was to develop a type of vector that could overcome the main barriers to non-viral gene delivery, which include cellular and nuclear membranes. With a deep understanding of the gene delivery process, it is acknowledged that efficient gene delivery requires multifunctional gene vectors that possess long circulation time, cellular or tissue targeting, and nuclear targeting. ${ }^{44}$ To overcome the barriers of cellular and nuclear membranes and achieve efficient gene therapy, a novel NLS conjugated cationic lipid (Dexa-LHON) was synthesized and used for the preparation of SLNs. A new, synthetic, Tf-containing ligand (Tf-PEG-PE) was used as a modifier, which was coated onto the nanocarrier's surface after the preparation of gene-loaded cationic SLNs (SLNs/pEGFP).

The investigation began with the synthesis of a novel NLS-conjugated cationic lipid (Dexa-LHON). GR is a nuclear receptor, and when it binds to the ligand, the receptor-ligand complex translocates from the cytoplasm

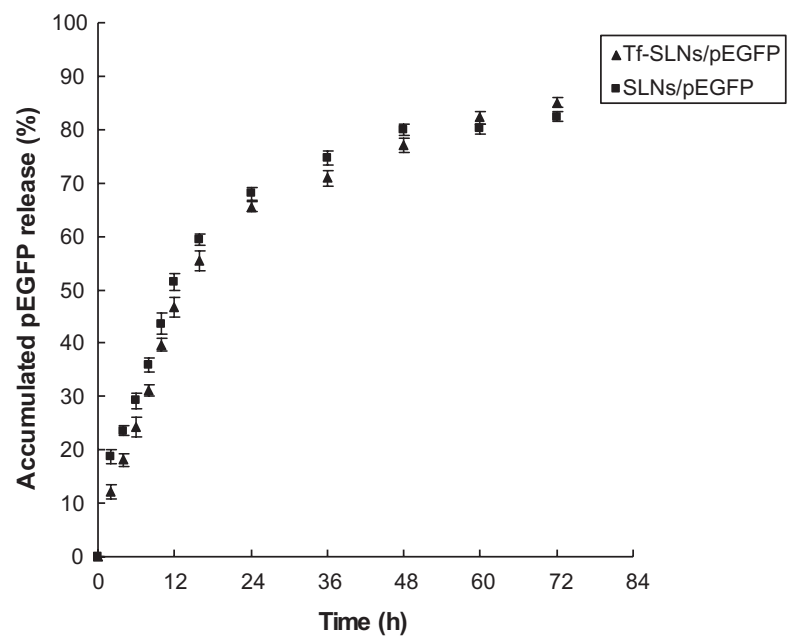

Figure 6 The in vitro release profiles of Tf-SLNs/pEGFP and SLNs/pEGFP. Abbreviations: Tf, transferrin; SLNs, solid lipid nanoparticles; pEGFP, enhanced green fluorescence protein plasmid. into the nucleus by means of the endogenous nuclear transport machinery. ${ }^{45}$ In addition, the nuclear pore is dilated up to $60 \mathrm{~nm}$ during this process to facilitate the uptake of transfected DNA into the nucleus. ${ }^{46}$ Moreover, glucocorticoids have a wide range of pharmacological effects, leading to pronounced anti-inflammatory and immunosuppressive effects and extensive clinical applications. Recent studies have proved that glucocorticoids encapsulated in longcirculating liposomes exert strong inhibitory effects on tumor growth in a low dose and low frequency schedule after intravenous administration. ${ }^{47-49}$ The results of those studies have confirmed the enhancement of gene delivery by using steroid polymers. In the present study, a Dexa-conjugated lipid (Dexa-LHON) was synthesized for the enhancement of nuclear localization of genes.

Tf is a blood plasma protein for iron ion delivery; it is a glycoprotein that binds iron very tightly, but reversibly. When Tf loaded with iron encounters Tf receptors (TfR) on the surface of a cell, they bind, and are consequently transported into the cell. Tf is especially useful in targeting cancer cells, as many cancer cells over-express TfR on their surface..$^{50} \mathrm{As}$ such, Tf was used as the target moiety, which could bind to the TfR on the HepG2 cells. A series of PEG-containing ligands, commonly named PEG-phosphatidylethanolamine (PEG-PE) conjugates were reported on and used for the modification of various vehicles to make active-targeting nanocarriers spontaneously accumulate in specific sites. ${ }^{51}$ Thus, PEG-PE was used at the anchor, and Tf-PEG-PE was used as the target ligand of the system.

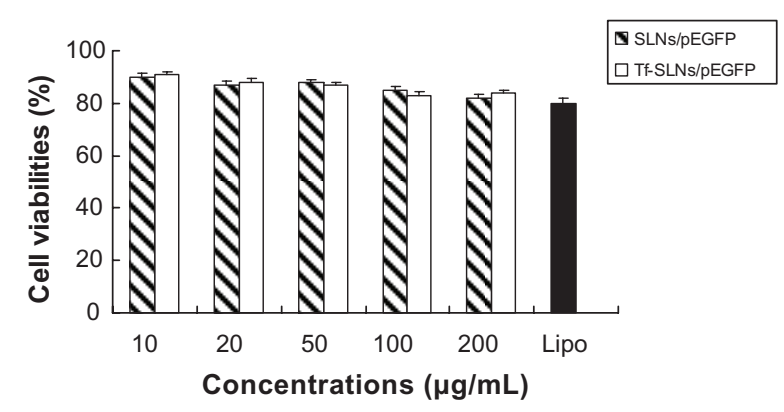

Figure 7 In vitro cytotoxicity evaluation of Tf-SLNs/pEGFP and SLNs/pEGFP. Abbreviations: Tf, transferrin; SLNs, solid lipid nanoparticles; pEGFP, enhanced green fluorescence protein plasmid. 


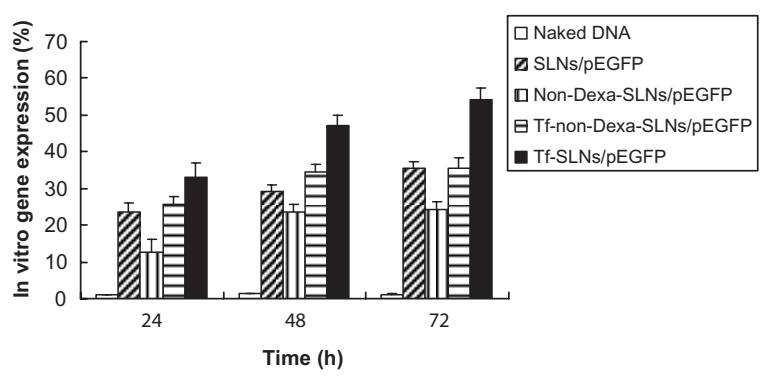

Figure 8 In vitro transfection efficiencies of different systems in HepG2 cells after being transfected for 24,48 , and 72 hours.

Abbreviations: Tf, transferrin; SLNs, solid lipid nanoparticles; pEGFP, enhanced green fluorescence protein plasmid; Dexa, dexamethasone.

In recent years, much attention has been paid to the use of cationic SLNs as gene carriers, which may offer a number of technological advantages, including better storage stability, relative ease to produce in large scale, and ability to be modified..$^{23}$ Our previous study focused on comparing two ways of modifying SLNs: pre-modification to form new modified vectors and post-modification after the gene was loaded. ${ }^{34}$ That investigation drew the conclusion that postmodification of gene-loaded SLNs has the advantage over pre-modified ones. Therefore, the post-modify method was carried out in the present study. During the modification procedure, Tf-PEG-PE ligands were extensively coated onto the SLNs/DNA surface, which covered their original surface charge and caused the decrease in zeta potential. The optimization of ligand-to-carrier ratio was carried out by measuring the zeta potential. The optimum ratio was obtained at 30\% (Tf-PEG-PE to SLNs/DNA, w/w) (Figure 5), and the optimum Tf-SLNs/pEGFP had a size of $221.8 \mathrm{~nm}$ and zeta potential of $+18.9 \mathrm{mV}$ (Table 1).

After the optimization of the modification procedure, the Tf-PEG-PE to SLNs/DNA ratio was determined as 30\%, and it was used in further experiments. A PicoGreen fluorometry assay was applied to determine the binding ability and in vitro release of Tf-SLNs/pEGFP and SLNs/pEGFP. The gene-loading efficiency of Tf-SLNs/pEGFP was $91 \%$, which was not significantly different from SLNs/pEGFP (90\%) (Table 1). The results demonstrated that binding the Tf-PEG-PE ligand did not detach the pEGFP from the complexes. The in vitro release profile (Figure 6) of TfSLNs/pEGFP showed a slightly slower release than SLNs/ pEGFP during the first 16 hours. This could be explained if the coating of the ligand hindered the release of DNA initially. After that, Tf-PEG-PE might detach from the vectors, allowing the DNA to release more freely. At the end of
$24 \mathrm{~h}$
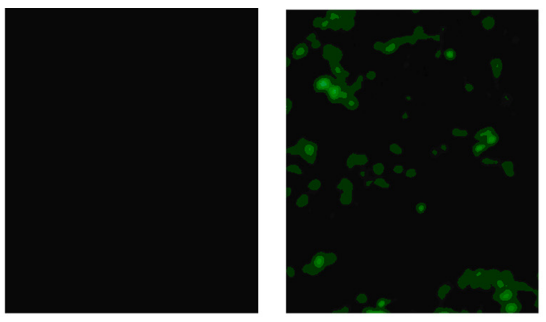

$48 \mathrm{~h}$
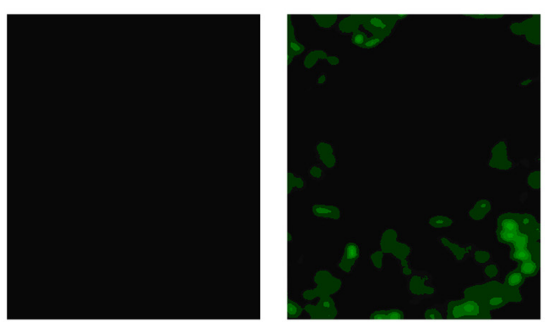

$72 \mathrm{~h}$

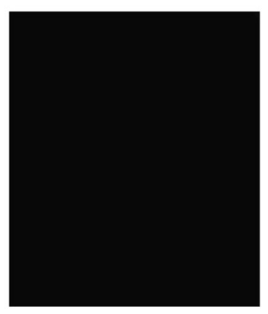

A

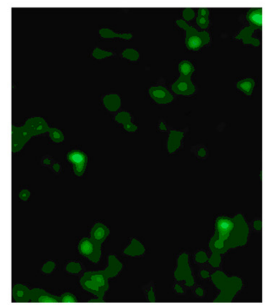

B
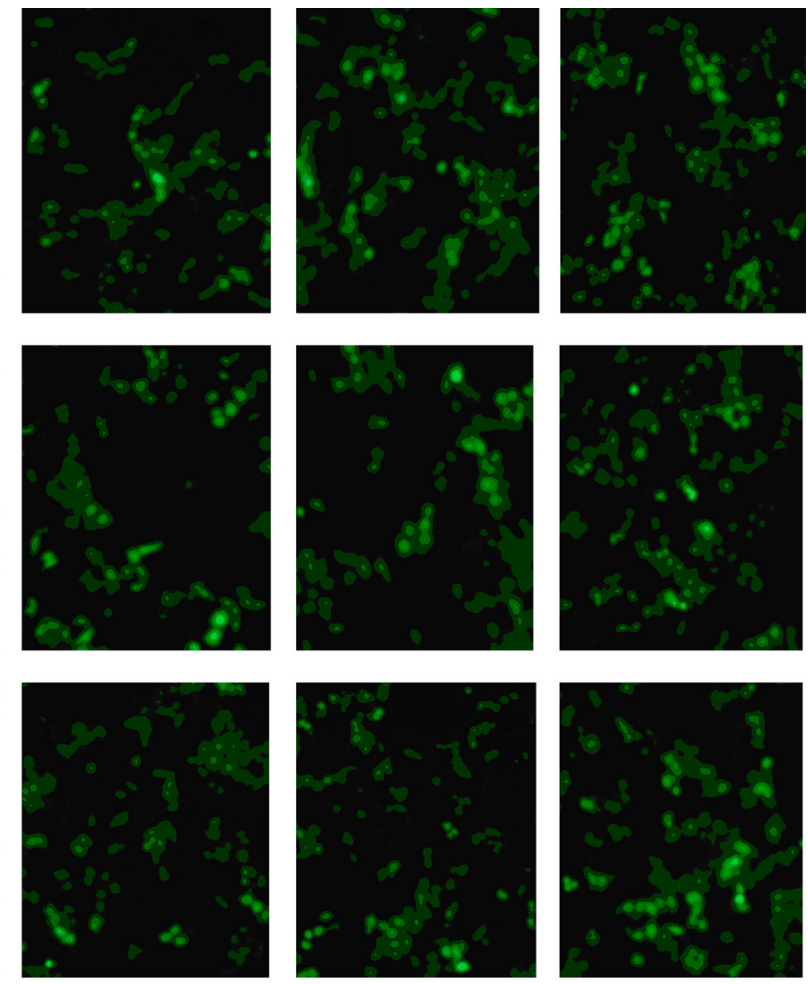

C

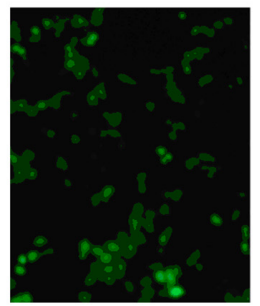

D

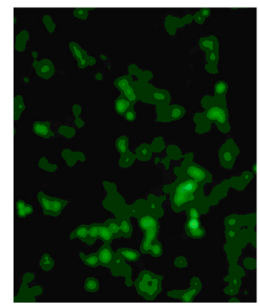

E

Figure 9 Fluorescent images of (A) naked DNA, (B) non-Dexa-SLNs/pEGFP, (C) unmodified SLNs/pEGFP, (D) Tf-non-Dexa-SLNs/pEGFP, and (E) Tf-SLNs/pEGFP at 24,48 , and 72 hours post-transfection.

Abbreviations: Tf, transferrin; SLNs, solid lipid nanoparticles; pEGFP, enhanced green fluorescence protein plasmid; Dexa, dexamethasone. 


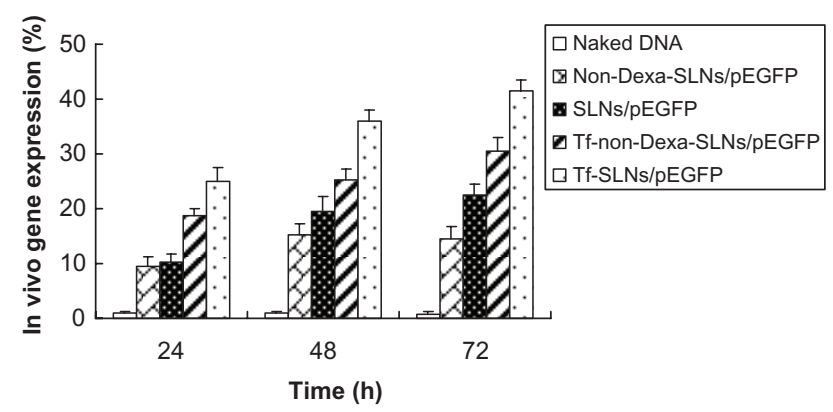

Figure 10 Quantitation of in vivo transfection efficiencies of Tf-SLNs/pEGFP and other vectors at 24,48 , and 72 hours post-transfection.

Abbreviations: Tf, transferrin; SLNs, solid lipid nanoparticles; pEGFP, enhanced green fluorescence protein plasmid; Dexa, dexamethasone.

the release study, the total amount of DNA delivered by the two types of vehicles was nearly the same (over $80 \%$ ).

In vitro cytotoxicity and transfection analysis were carried out in HepG2 cells. The cell viabilities of Tf-SLNs/pEGFP and SLNs/pEGFP over the studied concentration range were between $80 \%$ and $100 \%$, compared with the controls (Figure 7). Tf-SLNs/pEGFP showed no higher cytotoxicity than SLNs/pEGFP at all concentrations. When compared with naked DNA and SLNs/pEGFP, Tf-SLNs/pEGFP had greater transfection efficiency at all time points (Figure 8). This may be explained by the receptor-mediated active targeting mechanism. The Tf-SLNs/pEGFPs were more likely to bind to HepG2 cells via the TfR on the cells and thus, deliver DNA more easily into the cells. When compared with Tf-non-Dexa-SLNs/pEGFP (or non-Dexa-SLNs/pEGFP), TfSLNs/pEGFP (or SLNs/pEGFP) with Dexa-conjugated lipid carrier had higher transfection efficiency at all time points. This could be evidence that the presence of Dexa facilitates the binding of the vectors to the glucocorticoid receptor after cellular entry and translocates the complexes into the nucleus, resulting in increased gene expression.

The gene delivery abilities of Tf-SLNs/pEGFP were tested further in animal models. In vivo gene delivery and expression studies were evaluated against HepG2 tumor-bearing mice. After intravenous injection, the rats were euthanized and the HepG2 cells were isolated at 24, 48, and 72 hours and analyzed. Tf-SLNs/pEGFP displayed remarkably higher transfection efficiency than unmodified SLNs/pEGFP and the other vectors without Dexa at all time points, as shown in Figures 9 and 10. These results were in agreement with the in vitro data and could further ensure the cell targeting and nuclear targeting ability of Tf-SLNs/pEGFP mediated by Tf, Dexa, and their receptors. These observations strongly support the active targeting ability of Tf-PEG-PE modified SLNs/pEGFP, and the resulting vectors would be very useful for in vivo gene delivery.

\section{Conclusion}

This study showed that glucocorticoid-mediated targeting could successfully enhance gene expression in cancer cells. It also demonstrated that having a targeting ligand, such as TfPEG-PE, in SLNs formulations could significantly improve the transfection efficiency of the vector. Tf-SLNs/pEGFP had a remarkably higher transfection efficiency, both in vitro and in vivo, than unmodified SLNs/pEGFP and non-Dexa ones. It can be concluded, therefore, that $\mathrm{Tf}$ and Dexa can function as an excellent active targeting ligand to improve the cell and nuclear targeting ability of the carriers, and the resulting nanomedicine could be used as a promising active targeted drug/gene delivery system.

\section{Disclosure}

The authors report no conflicts of interest in this work.

\section{References}

1. Kong F, Zhou F, Ge L, Liu X, Wang Y. Mannosylated liposomes for targeted gene delivery. Int J Nanomedicine. 2012;7:1079-1089.

2. Kong F, Ge L, Liu X, Huang N, Zhou F. Mannan-modified PLGA nanoparticles for targeted gene delivery. Int J Photoenergy. 2012; Article ID 926754:7 pages. doi:10.1155/2012/926754.

3. Xie S, Pan B, Shi B, et al. Solid lipid nanoparticle suspension enhanced the therapeutic efficacy of praziquantel against tapeworm. Int $J$ Nanomedicine. 2011;6:2367-2374.

4. Ma K, Hu M, Qi Y, et al. Structure-transfection activity relationships with glucocorticoid-polyethyl-enimine conjugate nuclear gene delivery systems. Biomaterials. 2009;30(22):3780-3789.

5. Atkinson H, Chalmers R. Delivering the goods: viral and non-viral gene therapy systems and the inherent limits on cargo DNA and internal sequences. Genetica. 2010;138(5):485-498.

6. Choi JS, Ko KS, Park JS, Kim YH, Kim SW, Lee M. Dexamethasone conjugated poly(amidoamine) dendrimer as a gene carrier for efficient nuclear translocation. Int J Pharm. 2006;320(1-2):171-178.

7. Nishikawa M, Huang L. Nonviral vectors in the new millennium: delivery barriers in gene transfer. Hum Gene Ther. 2001;12(8):861-870.

8. Torchilin VP. Multifunctional nanocarriers. Adv Drug Deliv Rev. 2006;58(14):1532-1555.

9. Wagner E, Ogris M, Zauner W. Polylysine-based transfection systems utilizing receptor-mediated delivery. Adv Drug Deliv Rev. 1998; 30(1-3):97-113.

10. Ma K, Hu MX, Qi Y, et al. PAMAM-triamcinolone acetonide conjugate as a nucleus-targeting gene carrier for enhanced transfer activity. Biomaterials. 2009;30(30):6109-6118.

11. Lo WL, Chien Y, Chiou GY, et al. Nuclear localization signal-enhanced RNA interference of EZH2 and Oct4 in the eradication of head and neck squamous cell carcinoma-derived cancer stem cells. Biomaterials. 2012;33(14):3693-3709.

12. Yu VC, Naar AM, Rosenfeld MG. Transcriptional regulation by the nuclear receptor superfamily. Curr Opin Biotechnol. 1992;3(6): 597-602.

13. Adcock IM, Caramori G. Cross-talk between pro-inflammatory transcription factors and glucocorticoids. Immunol Cell Biol. 2001;79(4):376-384.

14. Shahin V,Albermann L, Schillers H, et al. Steroids dilate nuclear pores imaged with atomic force microscopy. J Cell Physiol. 2005;202(2): 591-601.

15. Strübing C, Clapham DE. Active nuclear import and export is independent of lumenal Ca2+ stores in intact mammalian cells. J Gen Physiol. 1999;113(2):239-248. 
16. Rebuffat A, Bernasconi A, Ceppi M, et al. Selective enhancement of gene transfer by steroid-mediated gene delivery. Nat Biotechnol. 2001;19(12):1155-1161.

17. Kim H, Bae YM, Kim HA, et al. Synthesis and characterization of dexamethasone-conjugated linear polyethylenimine as a gene carrier. $J$ Cell Biochem. 2010;110(3):743-751.

18. Kastrup L, Oberleithner H, Ludwig Y, Schafer C, Shahin V. Nuclear envelope barrier leak induced by dexamethasone. J Cell Physiol. 2006;206(2):428-434.

19. Kim H, Kim HA, Bae YM, Choi JS, Lee M. Dexamethasone-conjugated polyethylenimine as an efficient gene carrier with an anti-apoptotic effect to cardiomyocytes. J Gene Med. 2009;11(6):515-522.

20. Mi Bae Y, Choi H, Lee S, et al. Dexamethasone-conjugated low molecular weight polyethylenimine as a nucleus-targeting lipopolymer gene carrier. Bioconjug Chem. 2007;18(6):2029-2036.

21. Olbrich C, Bakowsky U, Lehr CM, Muller RH, Kneuer C. Cationic solid-lipid nanoparticles can efficiently bind and transfect plasmid DNA. J Control Release. 2001;77(3):345-355.

22. Vighi E, Ruozi B, Montanari M, Battini R, Leo E. Re-dispersible cationic solid lipid nanoparticles (SLNs) freeze-dried without cryoprotectors: characterization and ability to bind the pEGFP-plasmid. Eur J Pharm Biopharm. 2007;67(2):320-328.

23. Yu W, Liu C, Ye J, Zou W, Zhang N, Xu W. Novel cationic SLN containing a synthesized single-tailed lipid as a modifier for gene delivery. Nanotechnology. 2009;20(21):215102.

24. Yu W, Liu C, Liu Y, Zhang N, Xu W. Mannan-modified solid lipid nanoparticles for targeted gene delivery to alveolar macrophages. Pharm Res. 2010;27(8):1584-1596.

25. Lobovkina T, Jacobson GB, Gonzalez-Gonzalez E, et al. In vivo sustained release of siRNA from solid lipid nanoparticles. ACS Nano. 2011;5(12):9977-9983.

26. Doktorovova S, Shegokar R, Rakovsky E, et al. Cationic solid lipid nanoparticles (cSLN): structure, stability and DNA binding capacity correlation studies. Int J Pharm. 2011;420(2):341-349.

27. Oster CG, Kim N, Grode L, et al. Cationic microparticles consisting of poly(lactide-co-glycolide) and polyethylenimine as carriers systems for parental DNA vaccination. J Control Release. 2005;104(2):359-377.

28. Pinnaduwage P, Schmitt L, Huang L. Use of a quaternary ammonium detergent in liposome mediated DNA transfection of mouse L-cells. Biochim Biophys Acta. 1989;985(1):33-37.

29. Tabatt K, Sameti M, Olbrich C, Müller RH, Lehr CM. Effect of cationic lipid and matrix lipid composition on solid lipid nanoparticle-mediated gene transfer. Eur J Pharm Biopharm. 2004;57(2):155-162.

30. Tang F, Hughes JA. Synthesis of a single-tailed cationic lipid and investigation of its transfection. J Control Release. 1999;62(3):345-358.

31. Gilot D, Miramon ML, Benvegnu T, et al. Cationic lipids derived from glycine betaine promote efficient and non-toxic gene transfection in cultured hepatocytes. J Gene Med. 2002;4(4):415-427.

32. Lukyanov AN, Gao Z, Mazzola L, Torchilin VP. Polyethylene glycol-diacyllipid micelles demonstrate increased accumulation in subcutaneous tumors in mice. Pharm Res. 2002;19(10):1424-1429.

33. Torchilin VP. Recent advances with liposomes as pharmaceutical carriers. Nat Rev Drug Discov. 2005;4(2):145-160.
34. Jiang Z, Sun C, Yin Z, et al. A comparison of two kinds of nanomedicine for targeted gene therapy: Pre-modified or post-modified gene delivery systems. Int J Nanomedicine. 2012;7:2019-2031.

35. Singh M. Transferrin as a targeting ligand for liposomes and anticancer drugs. Curr Pharm Des. 1999;5(6):443-451.

36. Bellocq NC, Pun SH, Jensen GS, Davis ME. Transferrin-containing, cyclodextrin polymer-based particles for tumor-targeted gene delivery. Bioconjug Chem. 2003;14(6):1122-1132.

37. Wagner E, Curiel D, Cotton M. Delivery of drugs, proteins and genes into cells using transferrin as a ligand for receptor-mediated endocytosis. Adv Drug Deliv Rev. 1994;14:113-135.

$38 \mathrm{Li} \mathrm{H}$, Qian ZM. Transferrin/transferrin receptor-mediated drug delivery. Med Res Rev. 2002;22(3):225-250.

39. Maruyama K. Intracellular targeting delivery of liposomal drugs to solid tumors based on EPR effects. Adv Drug Deliv Rev. 2011;63(3): 161-169.

40. Jiang YY, Liu C, Hong MH, Zhu SJ, Pei YY. Tumor cell targeting of transferrin-PEG-TNF-alpha conjugate via a receptor-mediated delivery system: design, synthesis, and biological evaluation. Bioconjug Chem. 2007;18(1):41-49.

41. Liu C, Yu W, Chen Z, Zhang J, Zhang N. Enhanced gene transfection efficiency in CD13-positive vascular endothelial cells with targeted poly(lactic acid)-poly(ethylene glycol) nanoparticles through caveolaemediated endocytosis. J Control Release. 2011;151(2):162-175.

42. Suzuki R, Takizawa T, Kuwata Y, et al. Effective anti-tumor activity of oxaliplatin encapsulated in transferrin-PEG-liposome. Int J Pharm. 2008;346(1-2):143-150.

43. Li P, Liu D, Miao L, et al. A pH-sensitive multifunctional gene carrier assembled via layer-by-layer technique for efficient gene delivery. Int J Nanomedicine. 2012;7:925-939.

44. Kamiya H, Akita H, Harashima H. Pharmacokinetic and pharmacodynamic considerations in gene therapy. Drug Discov Today. 2003;8(21):990-996.

45. Pujols L, Mullol J, Roca-Ferrer J, et al. Expression of glucocorticoid receptor alpha- and beta-isoforms in human cells and tissues. Am J Physiol Cell Physiol. 2002;283(4):C1324-C1331.

46. Shahin V, Albermann L, Schillers H, et al. Steroids dilate nuclear pores imaged with atomic force microscopy. J Cell Physiol. 2005;202(2): 591-601.

47. Schiffelers RM, Metselaar JM, Fens MH, Janssen AP, Molema G, Storm G. Liposome-encapsulated prednisolone phosphate inhibits growth of established tumors in mice. Neoplasia. 2005;7(2):118-127.

48. Banciu M, Schiffelers RM, Fens MH, Metselaar JM, Storm G. Anti-angiogenic effects of liposomal prednisolone phosphate on B16 melanoma in mice. J Control Release. 2006;113(1):1-8.

49. Banciu M, Metselaar JM, Schiffelers RM, Storm G. Liposomal glucocorticoids as tumor-targeted anti-angiogenic nanomedicine in B16 melanoma-bearing mice. J Steroid Biochem Mol Biol. 2008;111(1-2): 101-110.

50. Yu W, Zhang N. Surface modification of nanocarriers for cancer therapy. Curr Nanosci. 2009;5(2):123-134.

51. Torchilin VP. Micellar nanocarriers: pharmaceutical perspectives. Pharm Res. 2007;24(1):1-16.
International Journal of Nanomedicine

\section{Publish your work in this journal}

The International Journal of Nanomedicine is an international, peerreviewed journal focusing on the application of nanotechnology in diagnostics, therapeutics, and drug delivery systems throughout the biomedical field. This journal is indexed on PubMed Central, MedLine, CAS, SciSearch $\AA$, Current Contents $\AA /$ Clinical Medicine,

\section{Dovepress}

Journal Citation Reports/Science Edition, EMBase, Scopus and the Elsevier Bibliographic databases. The manuscript management system is completely online and includes a very quick and fair peer-review system, which is all easy to use. Visit http://www.dovepress.com/ testimonials.php to read real quotes from published authors. 